BOOK REVIEW

\section{Debates and Dilemmas in Promoting Health: a Reader}

Edited by M Sidell, L Jones, J Katz, et al. 2nd edition: Palgrave Macmillan, 2003, pp 400. ISBN 0333694171

It is often said that the health promoter must be an expert in generalisation and Debates and Dilemmas in Promoting Health is an illustration of the vast range of issues influencing practice and policy health promotion today.

The publication is part of The Open University undergraduate course "Promoting Health: Skills, Perspectives and Practice". It presents a collection of articles by an impressive range of authors, and although the preface states that these aim to challenge preconceptions and evoke a critical understanding of current health issues, some of these are drawn from other publications and are some years old.

The book is divided into four sections and the introduction to each section from the editors attempts to summarise the key points and common themes. Overall, the reader should be left in no doubt about the complexity and lack of definitive answers in modern health promotion.

In the years since the defining events of the 1970s and 1980s that gave rise to the Alma Ata Declaration and the Ottawa Charter, health promotion has been increasingly subject to debates, dilemmas, and criticisms. The knowledge base for health advice becomes seemingly ever more complex and the balance between individual, community, and government responsibility appears to be in continual flux. The all-embracing definitions of health with which we work present enormous challenges in a climate of increasing need to demonstrate output and cost effectiveness.

The first section, "Key issues in health promotion", aims to explore some of the current thinking at the heart of health promotion, and offers a number of different perspectives on this thinking. Charles Webster and Jeff French provide a historical account of the development of health promotion and "The New Public Health" in the UK, and remind us that conflict and questioning date back to the early days of the public health movement.

Other chapters in this section examine different models of health and of health promotion, as well as explorations of lay perspectives and cross cultural interpretations of health. There are some gems in this collection including an essay from Hilary Graham, who guides the reader through the growing literature on inequalities in health and explores the individual, social, and geographical factors associated with inequality. Another is the chapter on social capital from Penelope Hawe and Alan Shiell, who also refer to the agenda for tacking inequalities in health and argue that the social capital literature could lead to more sophisticated (and less "individual-level") approaches to health promotion interventions that focus on settings.

The final article in section one is an essay by Ralph Lefebvre who argues that social marketing is misunderstood and has frequently been used by health promoters in a too limited way.

The second section deals with the evidence base for health promotion and examines some of debates about the nature of evidence and some of the ethical issues. For example, it includes examples that illustrate the need for caution in interpreting epidemiological data such as a chapter on the "French paradox" by Michael L Burr as well as a discussion on the limited conclusions that can be drawn on relationships when data are derived from different sources.

This section is not for the reader seeking practical advice on gathering evidence. It is not a guide to evaluation methodologies and when to use them, but an insight into the different types of evidence that health promoters may need to use. This may include data derived from disciplines as diverse as epidemiology, the social and behavioural sciences, clinical practice, and health economics. The challenge for the student is to grasp their relevant contributions and the limitations of these different methods.

A chapter by David McQueen and Laurie M Anderson discusses the nature of evidence. In today's strongly market driven health sector, the authors observe that the word itself is often at the very heart of current discussions in public health. They argue, however, that because "evidence" is closely associated with rigorous experimental designs, its use in health promotion is also questionable. Nevertheless, McQueen and Anderson conclude on an optimistic note, and comment that the emerging theoretical perspective of health promotion, embracing participation, context, and dynamism, is also influencing current thinking on evaluation design and methodologies. Thus, doors are opening to new methods, more appropriate indicators, and broader monitoring systems, reflecting a shift of focus towards population health and away from the individual.

Section three focuses on the wider context and discusses the influence of environmental, social, political, and economic factors on individual health. Herein lies one of the great dilemmas for health promoters, in that so many of the factors that influence health lie outside of the health sector and the control of the people who work in it. As a powerful illustration of this, the chapter by McMichael and Beaglehole discusses global trends such as changing labour markets, food production methods, broadening income differentials, environmental and climatic changes. Wendy Farrant, in "Pedogogy of the oppressed", writes about the community health movement in the UK and apparent government opposition at the end of the twentieth century to one of the core health promotion strategies-strengthening community action.

The role of healthy public policy is also critical in this debate, and contributions by Adrian Davis in Europe and Corti, Holman, and colleagues in Western Australia both illustrate the critical importance of policy change in creating healthier environments. A thought provoking contribution from Ron Labonte further explores this vital relation between health and environment and sets out some principles for decision making in order to achieve sustainable development.

The fourth and final section is on looking forward and offers a series of possible agendas for the 21 st century. Picking up some of the themes discussed earlier in the book, this includes revisiting issues such as inequalities in health and broader social changes, and explores some new visions and perspectives for the future.

Dan Beauchamp's chapter on "Lifestyle, public health and paternalism" raises the dilemma that a significant proportion of disease and ill health is related to personal risk taking and discusses the need to balance paternalism with self-determination within society as a whole. Other chapters touch on the rapidly growing "self-care" movement in the USA and the explosion of health information now accessible via the Internet. Several contributions explore specific examples of local approaches that hold promise for the future, such as the health promoting school model in the chapter by Susan Denman et al, and the proliferation of "area-based" initiatives in the UK such as Healthy Living Centres, Health Action Zones, and the "Sure Start" programme, described by Michael Kelly and Amanda Killoran. Echoing a continually recurring theme, Peter Townsend encourages us to "think globally, act locally" in the agenda for social justice and reducing inequality.

The final chapter, by Lowell S Levin and Erio Ziglio returns to the broad agenda and serves to remind the reader that health promotion needs to be positioned at the heart of social and economic development and should be perceived as a health investment strategy. They offer 10 essential requirements for "investment for health" encompassing political priority for health, accountability for health improvement across government, a greater understanding of health issues among the public and policy makers, and clearer incentives and goals.

\section{Modern health promotion involves more than simply handing out leaf- lets and putting up posters}

In summary, this publication sets out an impressive agenda that drives the reader through the plethora of current and emerging issues facing modern health promotion in the 21st century. Although some of the chapters are certainly more easily digestible than others, it will nevertheless provide a comprehensive insight for anyone wanting to learn more about this increasingly more complex field. No-one can be in any doubt after reading this publication that modern health promotion involves more than simply handing out leaflets and putting up posters.

If I have one small criticism, it is that as an update of an earlier edition published in 1997 and drawing on a number of articles derived from other sources, some of the content now appears a little dated. For example, there are references to surveys conducted over 10 years ago, and in these instances it is a pity the original authors were not invited to update their articles. Overall though, this is a thought provoking and informative book which provides a thorough examination of some of the most critical issues influencing health promotion today, and should be valuable for both students and experienced practitioners.

J Clarkson jclarkson@healthway.wa.gov.au 